\title{
In situ reprogramming to transdifferentiate fibroblasts into cardiomyocytes using adenoviral vectors: Implications for clinical myocardial regeneration
}

Megumi Mathison, MD, PhD, ${ }^{a}$ Vivek P. Singh, PhD, ${ }^{a}$ Maria J. Chiuchiolo, PhD, ${ }^{b}$ Deepthi Sanagasetti, MS, Yun Mao, MD, ${ }^{\text {a }}$ Vivekkumar B. Patel, MD, ${ }^{a}$ Jianchang Yang, MD, PhD, ${ }^{a}$ Stephen M. Kaminsky, PhD, Ronald G. Crystal, MD, ${ }^{\mathrm{b}}$ and Todd K. Rosengart, $\mathrm{MD}^{\mathrm{a}, \mathrm{c}}$

\section{ABSTRACT}

Objective: The reprogramming of cardiac fibroblasts into induced cardiomyocytelike cells improves ventricular function in myocardial infarction models. Only integrating persistent expression vectors have thus far been used to induce reprogramming, potentially limiting its clinical applicability. We therefore tested the reprogramming potential of nonintegrating, acute expression adenoviral (Ad) vectors.

Methods: Ad or lentivirus vectors encoding Gata4 (G), Mef2c (M), and Tbx5 (T) were validated in vitro. Sprague-Dawley rats then underwent coronary ligation and Ad-mediated administration of vascular endothelial growth factor to generate infarct prevascularization. Three weeks later, animals received Ad or lentivirus encoding G, M, or T (AdGMT or LentiGMT) or an equivalent dose of a null vector ( $\mathrm{n}=11,10$, and 10 , respectively). Outcomes were analyzed by echocardiography, magnetic resonance imaging, and histology.

Results: Ad and lentivirus vectors provided equivalent $\mathrm{G}, \mathrm{M}$, and T expression in vitro. AdGMT and LentiGMT both likewise induced expression of the cardiomyocyte marker cardiac troponin $\mathrm{T}$ in approximately $6 \%$ of cardiac fibroblasts versus $<1 \%$ cardiac troponin $\mathrm{T}$ expression in AdNull (adenoviral vector that does not encode a transgene)-treated cells. Infarcted myocardium that had been treated with AdGMT likewise demonstrated greater density of cells expressing the cardiomyocyte marker beta myosin heavy chain 7 compared with AdNulltreated animals. Echocardiography demonstrated that AdGMT and LentiGMT both increased ejection fraction compared with AdNull (AdGMT: 21\% \pm 3\%, LentiGMT: $14 \% \pm 5 \%$, AdNull: $-0.4 \% \pm 2 \% ; P<.05)$.

Conclusions: Ad vectors are at least as effective as lentiviral vectors in inducing cardiac fibroblast transdifferentiation into induced cardiomyocyte-like cells and improving cardiac function in postinfarct rat hearts. Short-term expression Ad vectors may represent an important means to induce cardiac cellular reprogramming in humans. (J Thorac Cardiovasc Surg 2017;153:329-39)

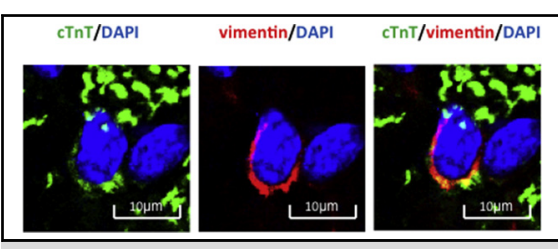

iCMs express the cardiomyocyte marker cTnT (green) and fibroblast marker vimentin (red).

\section{Central Message}

Adenoviral vectors reprogram cardiac fibroblasts into induced cardiomyocyte cells and improve cardiac function in the rat postinfarct heart.

\section{Perspective}

Cardiac reprogramming has thus far been accomplished by the use of only integrating chronic expression vectors such as retrovirus and lentivirus, potentially limiting the clinical applicability of this new myocardial regeneration strategy. If reprogramming could be achieved with nonintegrating, acute expression adenoviral vectors, the clinical applicability of cardiac cellular reprogramming would be enhanced greatly.

See Editorial Commentary page 340.

See Editorial page 327.

\footnotetext{
From the a Division of Cardiothoracic Surgery, Michael E. DeBakey Department of Surgery, Baylor College of Medicine; 'bepartment of Genetic Medicine, Weill Cornell Medical College, New York, NY; and ${ }^{\mathrm{c}}$ Department of Cardiovascular Surgery, Texas Heart Institute, Houston, Tex.

This study was funded by the National Heart Lung and Blood Institute (1R01HL121294-01A1 [to T.R.]) and supported in part by the BCM Cytometry and Cell Sorting Core (National Institutes of Health grants P30AI036211, P30CA125123, S10RR024574; National Center for Research Resources grant S10RR024574; National Institute of Allergy and Infectious Diseases grant AI036211; and National Cancer Institute grant P30CA125123).
}

M.M. and V.P.S. contributed equally to this article.

Received for publication March 22, 2016; revisions received Sept 13, 2016; accepted for publication Sept 15, 2016; available ahead of print Oct 20, 2016

Address for reprints: Todd K. Rosengart, MD, 1 Baylor Plaza, MS 390, Houston, TX 77030 (E-mail: todd.rosengart@bcm.edu).

$0022-5223 / \$ 36.00$

Copyright (C) 2016 by The American Association for Thoracic Surgery

http://dx.doi.org/10.1016/j.jtcvs.2016.09.041 


$\begin{aligned} & \text { Abbreviations and Acronyms } \\ & \text { Ad }=\text { adenoviral/adenovirus } \\ & \text { AdGMT }=\text { cocktail of adenovirus vectors } \\ & \text { expressing Gata4, Mef2c, or Tbx5 } \\ & \text { AdNull } \text { adenoviral vector that does not encode } \\ & \text { a transgene } \\ & \text { cTnT }=\text { cardiac troponin T } \\ & \text { EF }=\text { ejection fraction } \\ & \text { FACS }=\text { fluorescence-activated cell sorting } \\ & \text { GFP }=\text { green fluorescent protein } \\ & \text { iCM }=\text { induced cardiomyocyte-like cell } \\ & \text { iPS }=\text { induced pluripotent stem cell } \\ & \text { LentiGMT }= \text { cocktail of lentivirus vectors } \\ & \text { expressing gata4, Mef2c, or Tbx5 } \\ & \text { MRI }=\text { magnetic resonance imaging } \\ & \text { MYH7 }=\text { beta myosin heavy chain } 7 \\ & \text { OCT }=\text { optimum cutting temperature } \\ & \text { pu }=\text { particle unit } \\ & \text { TU }=\text { transducing unit } \\ & \text { VEGF }= \text { vascular endothelial growth factor }\end{aligned}$

Scanning this QR code will take you to supplemental figures for this article.

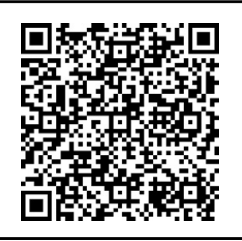

Cellular reprogramming represents a promising new potential treatment for cardiac failure, offering the possibility of regenerating myocardium from postinfarct scar tissue through the in situ conversion of (infarct-zone) fibroblasts into (induced) cardiomyocyte-like cells (iCMs). ${ }^{1-7}$ The premise for such cellular "transdifferentiation" was inspired by Takahashi and Yamanaka's ${ }^{8}$ induced pluripotent stem (iPS) cell studies but using instead a variety of (cardio) differentiating transcription factors and/or other agents to directly generate iCMs without iPS staging. ${ }^{1-7,9-15}$ After encouraging initial in vitro efforts, a number of groups, including our own, have demonstrated the efficacy of such transdifferentiation strategies in producing clinically relevant improvements in postinfarct ventricular function. ${ }^{2,16-20}$

The chronic expression (ie, retrovirus and lentivirus) gene transfer vectors used thus far to induce cellular reprogramming all integrate genes into host genomes, thereby posing potentially significant risks of mutagenesis that would limit their clinical applicability. ${ }^{21,22}$ Given that only a limited duration of transdifferentiating gene expression appears to be required to induce iPS and iCM generation, ${ }^{1,23}$ we hypothesized that (nonintegrating) acute expression vectors such as adenovirus (Ad) might represent an alternative, more clinically relevant cellular reprogramming vector. We consequently assessed the efficacy of Ad versus lentivirus vectors encoding Gata4, Mef2c, and Tbx5 to transdifferentiate cardiac fibroblasts into iCMs and thereby improve cardiac function in postinfarct models. Our findings provide suggest that reprogramming factors need be expressed only transiently to induce cellular reprogramming. Intriguingly, these data counter the long-held belief that Ad-infected cells are subject to immune-mediated cytolysis that would otherwise invalidate the option of an Ad-based cellular reprogramming strategy. ${ }^{24,25}$

\section{METHODS}

\section{Vectors and Cells}

A replication-deficient Ad vector expressing all 3 major isoforms of vascular endothelial growth factor (121, 165, and 189) was used to induce vascularization of infarcted myocardial tissues, as previously described. ${ }^{16,26}$ Lentivirus vectors encoding Gata4 (G), Mef2c (M), Tbx5 (T), or green fluorescent protein (LentiGFP) as well as an analogous Ad vector expressing GFP (AdGFP) were likewise prepared as previously described. ${ }^{16}$

Ad vectors expressing the $\mathrm{G}, \mathrm{M}$, and $\mathrm{T}$ transcription factors were prepared by the Belfer Gene Therapy Core Facility at Weill Cornell Medical College (New York, NY). To summarize in brief, the G, M, and $\mathrm{T}$ transcription factor cDNA sequences were cloned as single constructs into Ad vectors to originate the AdGATA4, AdTbx5, and AdMef2c vectors, respectively (Figure E1). An analogous construct with an expression cassette without translatable sequence (adenoviral vector that does not encode a transgene, or AdNull) was used as a control vector. Ad vectors were produced by propagation in HEK293 T cells, as previously described. ${ }^{27}$

For in vitro studies, adult cardiac fibroblasts were harvested from 8- to 10-week-old male Sprague-Dawley rats (Harlan Sprague-Dawley Inc, Indianapolis, Ind) by the use of standard cell isolation protocols ${ }^{28}$ that were approved by the Baylor College of Medicine Institutional Animal Care and Use Committee (Houston, Tex). All animals received humane care in compliance with the Guide for the Care and Use of Laboratory Animals. The cells were then seeded onto a 10 -cm dish with a total of $1 \times 10^{6}$ cells for fluorescence-activated cell sorting (FACS) analysis, a 6-well dish with a total of $1 \times 10^{5}$ cells/well for Western analysis, and onto a 24-well dish with a total of $1 \times 10^{4}$ cells/well for immunofluorescence studies.

\section{In Vitro Studies}

Rat cardiac fibroblasts were transduced with the aforementioned Ad or lentivirus vectors $\left(1 \times 10^{9}\right.$ particle unit $[\mathrm{pu}]$ or $1 \times 10^{5}$ transducing unit [TU], respectively) in culture for 48 hours in the presence of $1 \mathrm{mg} / \mathrm{mL}$ polybrene (EMD Millipore, Billerica, Mass). The cells were then treated with fresh media and allowed to transdifferentiate in a tissue culture incubator for 14 days. For immunofluorescence studies, these cells were blocked with 10\% goat serum (Santa Cruz Biotechnology, Santa Cruz, Calif) followed by treatment of $5 \%$ goat serum plus primary antibodies against cardiac troponin T (cTnT; Abcam) or $\alpha$-sarcomeric actinin (Abcam). Primary antibodies were labeled with fluorescent secondary (647 nm, Alexa Fluor; Invitrogen, Waltham, Mass), and fluorescence was visualized with a Ti-S inverted phase/fluorescent microscope with an SPOT cooled 2.0-megapixel digital microscope camera system (Nikon, Tokyo, Japan).

For FACS analysis, cells treated with the vectors described previously were washed with phosphate-buffered saline. The cells were then detached by Gibco trypsin-EDTA $(0.05 \%$; ThermoFisher Scientific, Waltham, Mass), pelleted, and permeabilized with $0.1 \%$ saponin for 10 minutes at 
room temperature. The cells were then resuspended in anti-cTnT primary antibody solution containing $5 \%$ goat serum. After treatment with a fluorescent secondary antibody ( $647 \mathrm{~nm}$, Alexa Fluor; Invitrogen), the cells were fixed with $1 \%$ paraformaldehyde and analyzed on a FACSCalibur flow cytometer (Becton Dickinson, Franklin Lakes, NJ) using the FACSDIVA Software, version 6.0 (BD Biosciences, San Jose, Calif).

\section{Ad Persistence Study in Normal Adult Rat}

Eight- to ten-week-old adult male Sprague-Dawley rats (Harlan) were sedated, intubated, and connected to a ventilator as previously described. ${ }^{16}$ AdGFP $\left(1 \times 10^{9} \mathrm{pu}\right)$ or LentiGFP $\left(1 \times 10^{5} \mathrm{TU}\right)$ was injected directly into the left ventricular wall through left thoracotomy ( 5 injections in anterolateral area, each $20 \mu \mathrm{L}$, total volume, $100 \mu \mathrm{L}),{ }^{16}$ and 3 days or 14 days later, the hearts were harvested and embedded into optimum cutting temperature (OCT) compound (Sakura Finetek, Torrance, Calif), as described in the section "Histologic Examination." Visualization of green fluorescence under light microscopy was used to determine the persistence of transgene expression ( $\mathrm{n}=3$ per each group).

\section{Rat Coronary Artery Ligation Myocardial Infarction Model}

Myocardial infarction was created in adult male Sprague-Dawley rats $(\mathrm{n}=36)$ by coronary artery ligation ${ }^{16}$ via the use of protocols approved by the Baylor College of Medicine Institutional Animal Care and Use Committee. Four animals (11\%) died after the coronary ligation. Three weeks after coronary ligation and direct myocardial administration of Ad- vascular endothelial growth factor (VEGF; $1 \times 10^{9} \mathrm{pu}$ ), a cocktail of Ad or lentiviruses encoding Gata4, Mef2c, or Tbx5 (AdGMT or LentiGMT, respectively), or AdNull $\left(1 \times 10^{9}\right.$ pu for AdGMT and AdNull, $1 \times 10^{5}$ TU for LentiGMT) was administered by direct myocardial injection, as previously described. ${ }^{16}$ The rats were euthanized 4 weeks later by deep $(4 \%)$ isoflurane anesthesia followed by exsanguination, consistent with the guidelines of the American Veterinary Medical Association.

\section{Echocardiography}

Echocardiography was performed under light anesthesia with 3\% isoflurane using a Veno 770 Imaging System (VisualSonics, Inc, Toronto, Canada). ${ }^{16}$ Echocardiographic images were obtained in the parasternal long-axis and short-axis views at specified points by investigators who were unaware of the treatment groups. The left ventricular end-systolic and end-diastolic diameters were measured from the M-mode tracings. The change in the ejection fraction (EF) from baseline (day 21; immediately before reprogramming vector administration) to the final evaluation time point 4 weeks later (day 49) was calculated as: ([EF at day 49$]-[$ EF at day 21])/(EF at day 21).

\section{Magnetic Resonance Imaging (MRI)}

For MRI analyses, rats were sedated with $2 \%$ to $3 \%$ isoflurane and placed in a specially built holder insertable within the MR resonator and incorporating an animal telemetry (SA Instruments, Stony Brook, NY). MRIs were acquired with a 9.4 T, Bruker Avance III Biospec Spectrometer, 21-cm bore horizontal scanner with a 72-mm volume resonator (Bruker Bio Spin Corp, Billerica, Mass). Gated images were acquired at end-diastole and end-systole by the use of Intragate (Bruker BioSpin Corp, Billerica, Mass). Multislice axial scans were used to visualize the left and right ventricles and volumes analyzed with image processing software (Osirix, Bernex, Switzerland) to summate left and right ventricular space pixels and the known volume size of each pixel.

\section{Histologic Examination}

Rat hearts were prepared for histologic analysis as previously described. ${ }^{16}$ Infarct fibrosis was assessed using Masson trichrome staining of 7 sections per animal ( $n=5$ per group). The fibrotic area (blue) and the nonfibrotic region (red) were outlined in a blinded fashion with Adobe Photoshop Elements 9 software (Adobe, San Jose, Calif) and then quantified with MATLAB and Simulink software (MathWorks, Inc, Natick, Mass). The ventricular fibrosis percentage was calculated as: ([blue pixels/ \{blue + red pixels $\}] \times 100$ ).

For cardiomyocyte marker assays, one section per animal was stained with a primary antibody against beta myosin heavy chain 7 (anti-MYH7; Sigma-Aldrich, St. Louis, Mo) and then incubated with a secondary immunoglobulin $\mathrm{G}$ antibody. Three randomly selected microscopic fields per slide (at the center of the infarction zone, and in the mid areas between the center of infarction and the border zone [left and right]) were viewed at $200 \times$ magnification and graded semiquantitatively to determine $\mathrm{MYH}^{+}$ cell density. "High" MYH7 cell density was quantified based on the percentage of examined microscopic fields demonstrating at least $50 \%$ occupancy by cells stained for MYH7, as we have previously described. ${ }^{16}$

For immunofluorescence analysis, the heart was perfused with heparin $(5000 \mathrm{U} / \mathrm{mL})$ containing phosphate-buffered saline, sectioned as noted previously, and then immediately placed in OCT compound (Sakura Finetek); or snap frozen for GFP injection group. For cTnT and vimentin staining, hearts were perfused with the aforementioned buffer and then with $4 \%$ paraformaldehyde. After the hearts were excised, they were immersed in $30 \%$ sucrose solution, followed by overnight incubation at $4^{\circ} \mathrm{C}$, and then frozen. ${ }^{29}$

Frozen sections $(5 \mu \mathrm{m})$ were prepared from the OCT-embedded tissues. The slides were fixed and permeabilized in BD Cytofix/Cytoperm solution (BD Biosciences), then washed with BD Perm/Wash buffer 3 times and blocked with $10 \%$ donkey serum for 1 hour at room temperature. cTnT and vimentin (Abcam) primary antibodies were incubated overnight at $4^{\circ} \mathrm{C}$, followed by 3 washes, and then probed with fluorescent labeled secondary antibodies for 1 hour at room temperature. The slides were mounted with Dako Fluorescence Mounting Medium (Dako North America, Carpinteria, Calif) and observed with a Leica SP5 confocal microscope and LAS AF lite software (both from Leica Biosystems, Richmond, Ill).

\section{Statistical Analysis}

Statistical analysis was performed with SAS, version 9.2 (SAS Institute, Inc, Cary, NC). Data are presented as the mean \pm standard error of the mean, unless otherwise indicated. The normality of the data was first examined with a Shapiro-Wilk test. If the data had normal distribution, analysis of variance was used. If the data did not meet normality assumption, a Kruskal-Wallis test was used. MYH7-positive cell comparison was performed with the Fisher exact test. For the mean of the change in EF for each rat from the interval from the second surgery to assessment 4 weeks later, a Kruskal-Wallis test was carried out, followed by Bonferroni correction for Wilcoxon rank test.

A power analysis was conducted to determine appropriate animal sample size. On the basis of our previous experiments, ${ }^{16,17}$ total sample size was calculated as 30 for 3 groups, assuming a group standard deviation of 10 , alpha of 0.05 , and nominal power of $80 \%$. Because we anticipated mortality as about $15 \%$, we enrolled a total of 36 animals.

\section{RESULTS}

\section{Reprogramming Vector Validation Studies}

The competency of Ad vectors to induce the expression of Gata4 (G), Mef2c (M), and Tbx5 (T) in vitro was confirmed by Western analysis, which demonstrated GMT expression equivalent to that yielded by lentiviral vectors (Figure 1). The ability of these vectors to generate iCMs from cardiac fibroblasts in vitro was validated by detection of the cardiomyocyte-specific markers cTnT and $\alpha$-sarcomeric actinin, which were detected at 

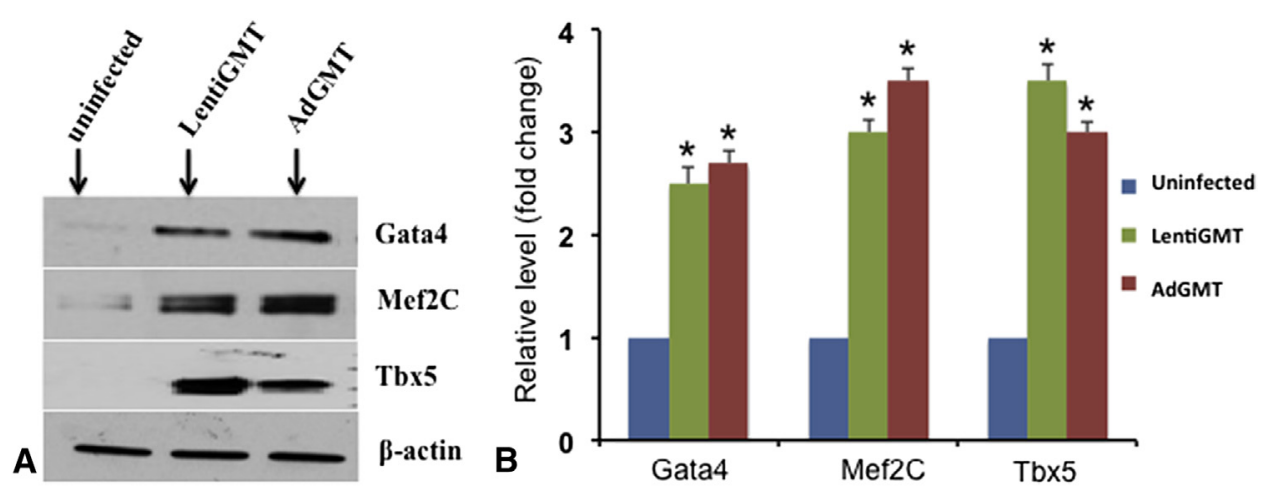

FIGURE 1. In vitro validation of vector expression. A, Western analysis for expression of Gata4, Mef2c, and Tbx5 after infection of adult rat cardiac fibroblasts with AdGMT or LentiGMT, as described in the Methods. Equivalent expression of Gata4, Mef2c, and Tbx5 is demonstrated by the AdGMT versus the LentiGMT vector ( $\mathrm{n}=3$ per group). B, Quantitative analyses for Gata4, Mef2C, and Tbx5 protein expression treated with Lenti-GMT and Ad-GMT viruses. ${ }^{*} P<.05$ versus uninfected. LentiGMT signifies 3 singlets, LentiGata4, LentiMef2c, and LentiTbx5, and AdGMT signifies 3 singlets, AdGata4, AdMef2c, and AdTbx5. LentiGMT, Cocktail of lentivirus vectors expressing gata4, Mef2c, or Tbx5; AdGMT, cocktail of adenovirus vectors expressing Gata4, Mef2c, or Tbx5.

approximately equivalent levels of expression by immunocytochemistry 14 days after AdGMT or LentiGMT transduction (Figure 2). These cardiomyocyte markers were not detected after cardiac fibroblast infection with AdNull. Consistent with these findings, FACS analysis demonstrated significantly increased cTnT expression after cardiac fibroblast treatment with AdGMT and LentiGMT compared with treatment with an AdNull control vector $(6.9 \% \pm 0.7 \%$ and $5.9 \% \pm 0.6 \%$ vs $0.6 \% \pm 0.1 \%$, respectively, both $P<.05$ ) (Figure E2).

Validation of the activity of our adenoviral and lentivirus vector constructs was further confirmed by detecting upregulation of the expression of Gata4, Mef2c, or Tbx5 in vivo after the administration of relevant vectors in our coronary ligation model. Expression levels of these were measured at those time points corresponding to peak expression for each vector; namely, at 3 days postadministration for Ad and at the time of terminal myocardial sampling 4 weeks after vector administration for lentivirus (Figure E3).

\section{Persistence of Ad-Infected Cells in Vivo}

To evaluate the persistence of Ad-infected myocardial cells in vivo, Ad or lentivirus vectors encoding the marker gene GFP were administered into naïve (nonligated) rat hearts. The number of cells expressing GFP was significantly greater 3 days after AdGFP versus LentiGFP administration (Figure 3,A), consistent with the known expression profiles of these 2 vectors. An equivalent number of cells expressed GFP 14 days after AdGFP versus LentiGFP administration, which represented a significant decrease in GFP expression at 14 days versus 3 days after AdGFP administration (Figure 3, $B$ ).

\section{Histologic Assessment of iCM Generation}

The extent of postinfarct left ventricular fibrosis measured as a percentage of the left ventricular wall area was decreased in hearts 4 weeks after AdGMT compared with AdNull administration $(6 \% \pm 1 \%$ vs $111 \% \pm 3 \%$, respectively; Figure E4), although this difference did not reach statistical significance. Nearly half of all randomly selected microscopic fields selected from this infarction area demonstrated a high density of cells $(>50 \%$ of the microscopic field) expressing the cardiomyocyte marker MYH7 after treatment of infarcted myocardium with AdGMT in our coronary ligation model (Figure 4). In comparison, none of the sections of hearts administered AdNull demonstrated a high density of cells expressing MYH7. LentiGMT-treated animals appeared to have fewer fields with high $\mathrm{MYH}^{+}$cell density compared with AdGMT-treated animals.

As further evidence of iCM generation in vivo, cells expressing both the cardiomyocyte marker cTnT and the fibroblast marker vimentin were identified in both AdGMT- and LentiGMT-treated hearts, suggesting the fibroblast origin of these MYH7 positive (iCM) cells (Figure 5). Such dually staining cells were not found in AdNull-treated hearts.

\section{Improvement in Ventricular Function After GMT Administration}

Echocardiographic analyses performed before and immediately after coronary artery ligation demonstrated that $\mathrm{EF}$ was reduced by $\sim 25 \%$ from baseline values after coronary ligation (Figure 6, A). This decrease in cardiac function persisted 3 weeks later, at the time of AdGMT, LentiGMT, or AdNull administration. The mean EF 4 weeks after AdGMT, LentiGMT, or AdNull administration, as measured by echocardiography and MRI, was greatest for animals receiving AdGMT (Table 1, Figure 7), although differences between groups did not achieve statistical significance as absolute values. The mean change in EF from the time of reprogramming factor administration to 

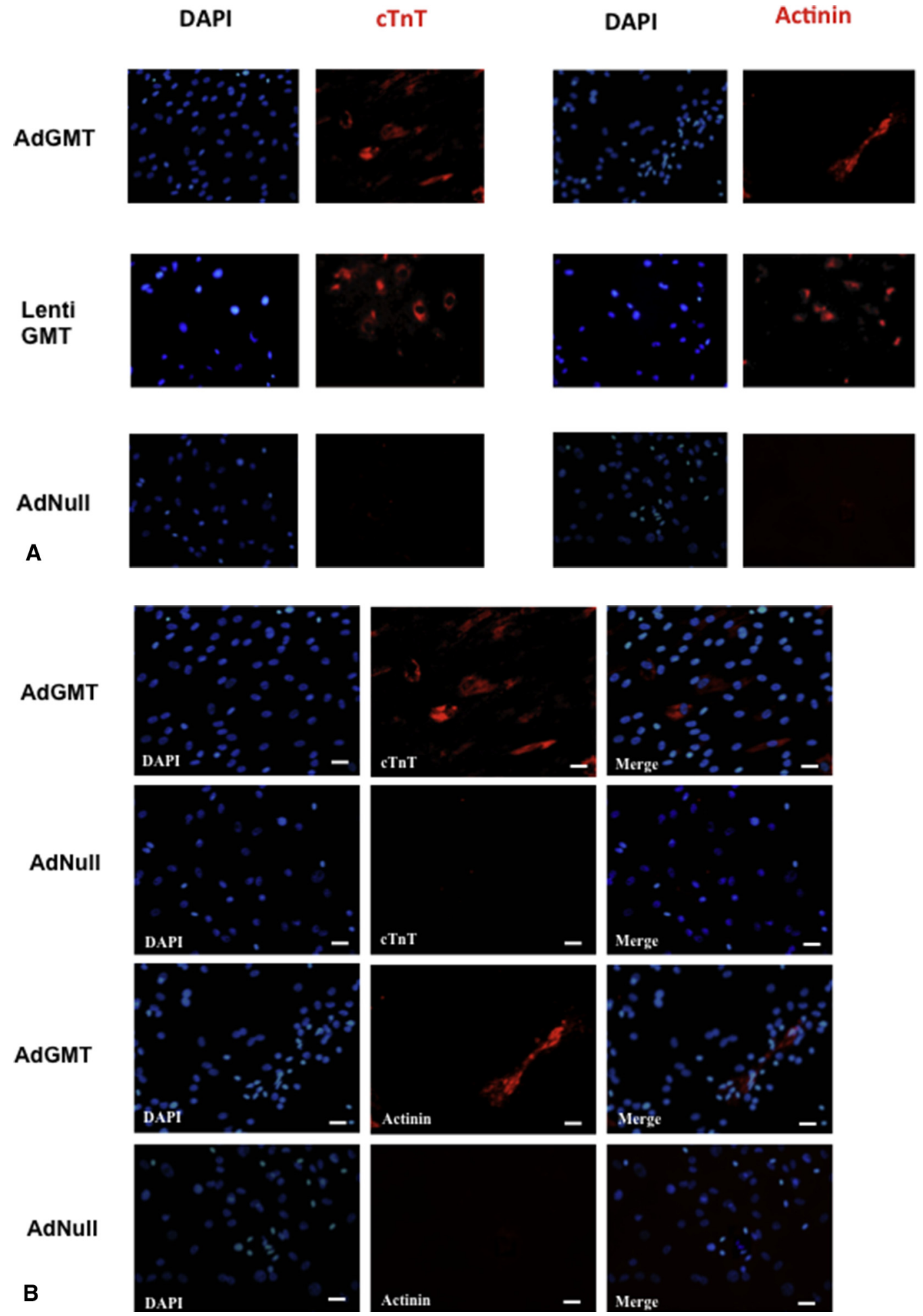

FIGURE 2. In vitro validation of iCM generation. Immunofluorescence analysis performed 14 days after infection of adult rat cardiac fibroblasts with AdGMT, LentiGMT, or AdNull demonstrated expression of the cardiomyocyte markers cTnT and $\alpha$-sarcomeric actinin (red staining) after GMT administration, but not after treatment with AdNull. A, First column represents DAPI staining to identify cell nuclei. B, Immunofluorescence analysis is presented with merged images after AdGMT versus AdNull treatment. First column represents DAPI staining to identify cell nuclei. Second column represents red staining of cardiomyocyte marker cTnT and $\alpha$-sarcomeric actinin. Third column depicts merge of previous 2 images. LentiGMT signifies 3 singlets, LentiGata4, LentiMef2c, and LentiTbx5, and AdGMT signifies 3singlets, AdGata4, AdMef2c, and AdTbx5. DAPI, 4',6-diamidino-2phenylindole; $c T n T$, cardiac troponin T; AdGMT, cocktail of adenovirus vectors expressing Gata4, Mef2c, or Tbx5; LentiGMT, cocktail of lentivirus vectors expressing gata4, Mef2c, or Tbx5; AdNull, adenoviral vector that does not encode a transgene. 

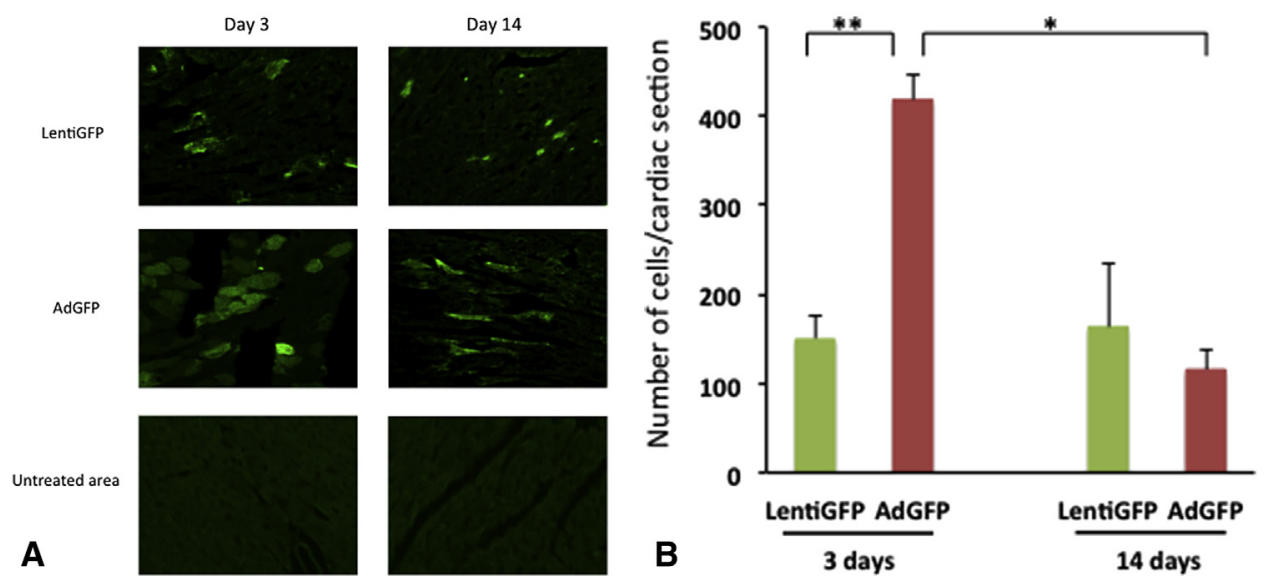

FIGURE 3. Adenovirus-infected cell persistency. Adult rats received AdGFP $(1 \times 109 \mathrm{pu})$ or LentiGFP $\left(1 \times 10^{5} \mathrm{TU}\right)$ injections into the heart, and 3 days or 14 days later, the hearts were harvested and embedded into OCT compound, as described in the Methods. A, Photomicrographs (200×) display representative GFP-positive cells in treated versus untreated myocardium. B, Quantification of cells with positive GFP is shown ( $\mathrm{n}=3$ per group). ${ }^{*} P<.05,{ }^{*} P<.01$. LentiGFP, Lentivirus green fluorescent protein; AdGFP, Ad vector expressing green fluorescent protein.

the time of final analysis 4 weeks later was significantly greater $(P<.05)$ after AdGMT and LentiGMT versus AdNull administration $(21 \% \pm 3 \%$ vs $14 \% \pm 5 \%$ and $-0.4 \% \pm 2 \%$, respectively; Figure $6, B$ ).

\section{DISCUSSION}

The present study demonstrates that cardiac cellular transdifferentiation and the generation of iCMs induced by Ad-mediated transfer of the reprogramming factors Gata4, Mef2c, and Tbx5 are associated with enhancement of postinfarction ventricular function in vivo. The use of Ad as a gene transfer vector has been shown in a wide variety of clinical trials to be well tolerated and effective in inserting transgenes of interest into host cells to induce these cells to express a secreted protein product as a therapeutic "drug." ${ }^{30}$ The use of gene transfer vectors for the purpose of cellular reprogramming differs significantly, however, from previous gene transfer strategies in that the host cell itself (the induced cardiomyocyte), rather than its secreted protein products, is thought to represent the therapeutic effector. In this context, the data from the current study are intriguing, especially in that they suggest a possible contradiction to long-held (but, to our knowledge, unproven) beliefs that immunity-mediated cytolytic elimination of Ad-infected cells by the host represents an important mechanism responsible for the transient duration of Ad transgene expression. ${ }^{24,25,31}$ Our observation of diminished but persistent cellular expression of the marker gene GFP at least 2 weeks after Ad vector administration is consistent with this premise and suggests that mechanisms other than host cell cytolysis, for example, innate cell immunity, may be responsible for the extinction of $\mathrm{Ad}$ transgene expression activity.

The ability of Ad and lentivirus vectors to overexpress the GMT transgenes in vitro and in vivo, as validated in the current study, is consistent with our previous reports describing the in vitro and myocardial expression
AdGMT

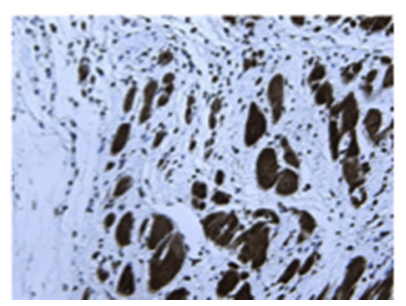

LentiGMT

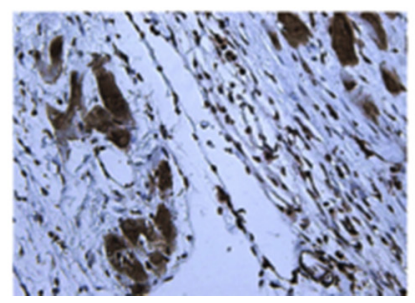

AdNull

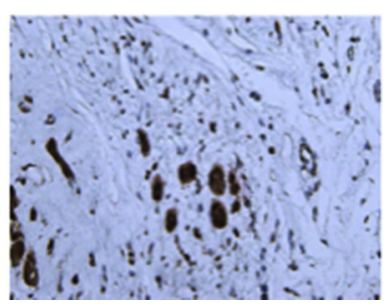

FIGURE 4. Cardiomyocyte density in infarct zones. The cardiomyocyte-specific marker MYH7 was detected in sections of myocardium harvested 4 weeks after the administration of AdGMT, LentiGMT, or AdNull (7 weeks after coronary ligation and administration of AdVEGF). Photomicrographs of representative sections of infarct zones from animals $(200 \times)$ treated with AdGMT (left), LentiGMT (middle), or AdNull (right) ( $\mathrm{n}=4$ per group). LentiGMT signifies 3 singlets, LentiGata4, LentiMef2c, and LentiTbx5, and AdGMT signifies 3 singlets, AdGata4, AdMef2c, and AdTbx5. AdGMT, Cocktail of adenovirus vectors expressing Gata4, Mef2c, or Tbx5; LentiGMT, cocktail of lentivirus vectors expressing gata4, Mef2c, or Tbx5; AdNull, adenoviral vector that does not encode a transgene. 


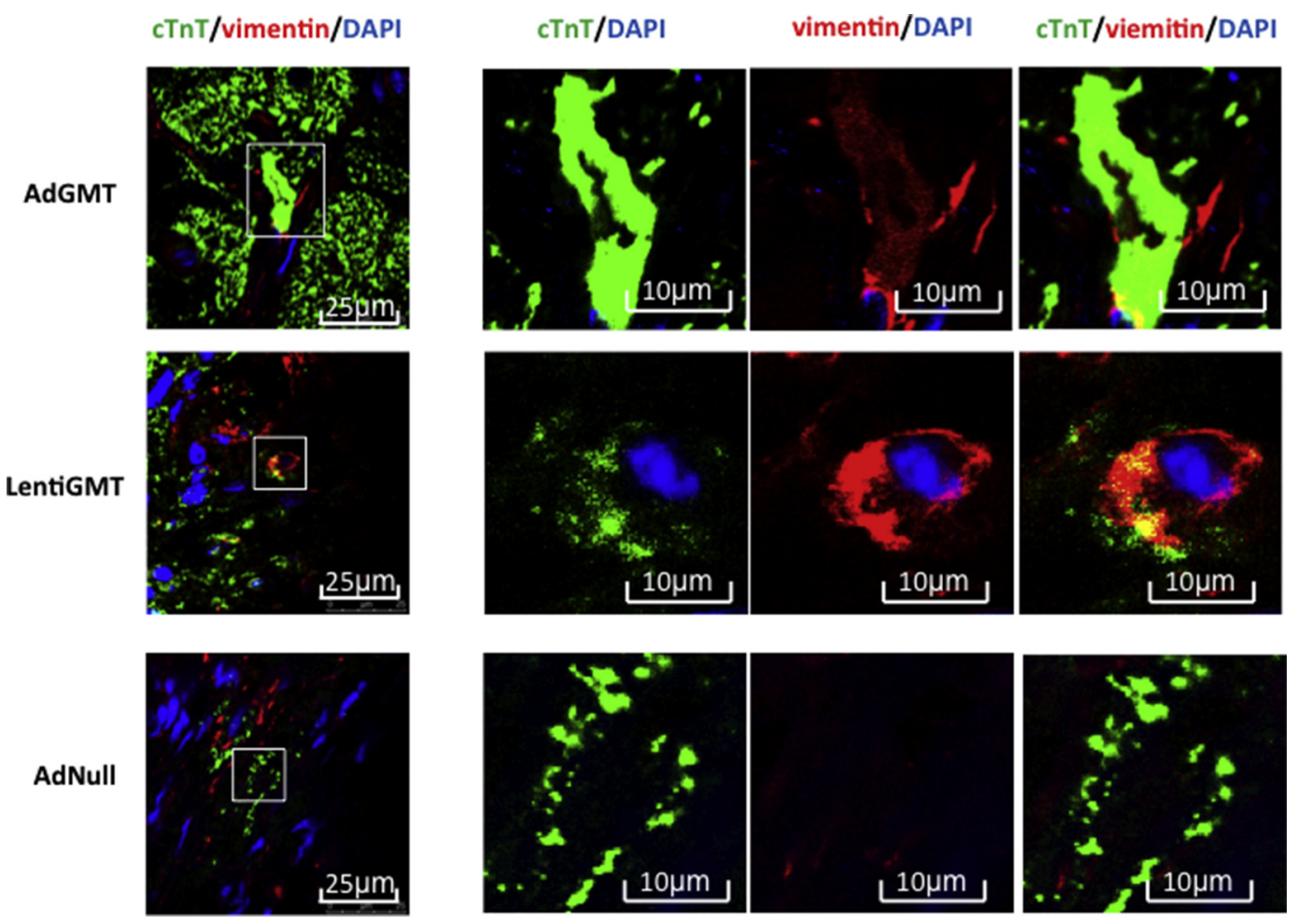

FIGURE 5. iCM detection after GMT treatment in vivo. Immunofluorescent staining of infarct zones for the cardiomyocyte marker cTnT (green), the fibroblast marker vimentin ( red), and the nuclear stain DAPI (blue) was performed after treatment of infarcted myocardium in vivo with AdGMT, LentiGMT, or AdNull, as described in the Methods. Photomicrographs depict cells expressing both cTnT and vimentin after AdGMT and LentiGMT treatment (yellow), suggesting the fibroblast origin of these cTnT-positive (iCM) cells. Coexpression of cTnT and vimentin was not detected in AdNull-treated hearts. Boxed area indicates region of greater magnification $(\mathrm{n}=2$ per group). LentiGMT signifies 3 singlets, LentiGata4, LentiMef2c, and LentiTbx5, and AdGMT signifies 3 singlets, AdGata4, AdMef2c, and AdTbx5. cTnT, Cardiac troponin; DAPI, 4',6-diamidino-2-phenylindole; AdGMT, cocktail of adenovirus vectors expressing Gata4, Mef2c, or Tbx5; LentiGMT, cocktail of lentivirus vectors expressing gata4, Mef2c, or Tbx5; AdNull, adenoviral vector that does not encode a transgene.

profiles of these vectors. ${ }^{16,17,32}$ Direct, "head-to-head" comparisons of transgene expression yielded by these 2 vectors are precluded by the different expression profiles of these vectors (ie, acute, early peaking expression with $\mathrm{Ad}$; sustained, late peaking expression with lentivirus). Nevertheless, both the expression data reported and the significant changes in infarct morphology and myocardial function after both lentiviral and adenoviral mediated transfer of GMT in our coronary ligation model support the in vivo efficacy of these vectors in inducing cellular reprogramming.

The increased infarct cardiomyocyte density and improvements in hemodynamic function that were observed well beyond the 1- to 2-week transgene expression interval classically associated with Ad vectors also reconfirm previous data suggesting that reprogramming factors need be present for only a limited duration of time-not more than several weeks-to induce sustained cellular transdifferentiation. ${ }^{1,23,31}$ The relative absence of such factors in mature cardiomyocytes after initial embryonic development and the reported effects of residual reprogramming gene expression in preventing the developmental competence of iPS cells further suggest that unnecessarily prolonged cell exposure to reprogramming factors confers no advantage in cell differentiation. ${ }^{33}$

In the context of the favorable hemodynamic effects provided by adenoviral compared with lentivirusmediated reprogramming factor delivery in the present study, the transient reprogramming conferred by $\mathrm{Ad}$ vectors might thus provide a beneficial, more "natural" recapitulation of native developmental processes compared with the prolonged reprogramming factor exposure provided by chronic expression vectors. These considerations are further supported by the potential host cell mutagenesis and oncogenicity risks associated with the host cell genome integration by virtue of which previously tested (retrovirus and lentivirus) chronic expression vectors induce prolonged transgene expression. ${ }^{21,22}$ Given the potential benefits of the use of Ad vectors, we plan long-term studies to further test both the persistence of the Ad genome and the sustainability of Ad-mediated cardiac cellular reprogramming in the context of diminished Ad transgene expression. 
AdGMT

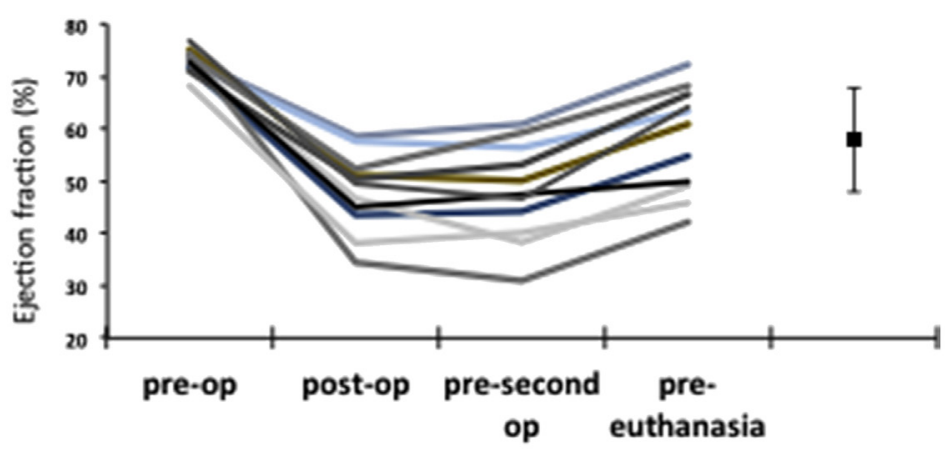

LentiGMT

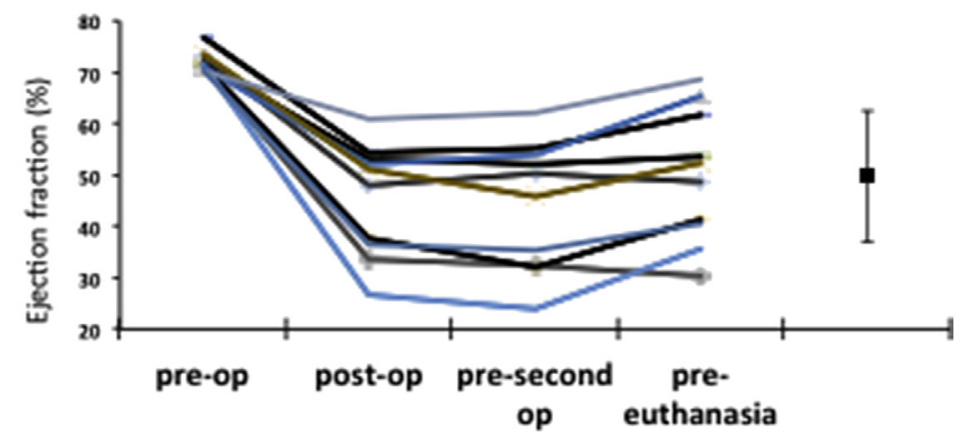

AdNull

A
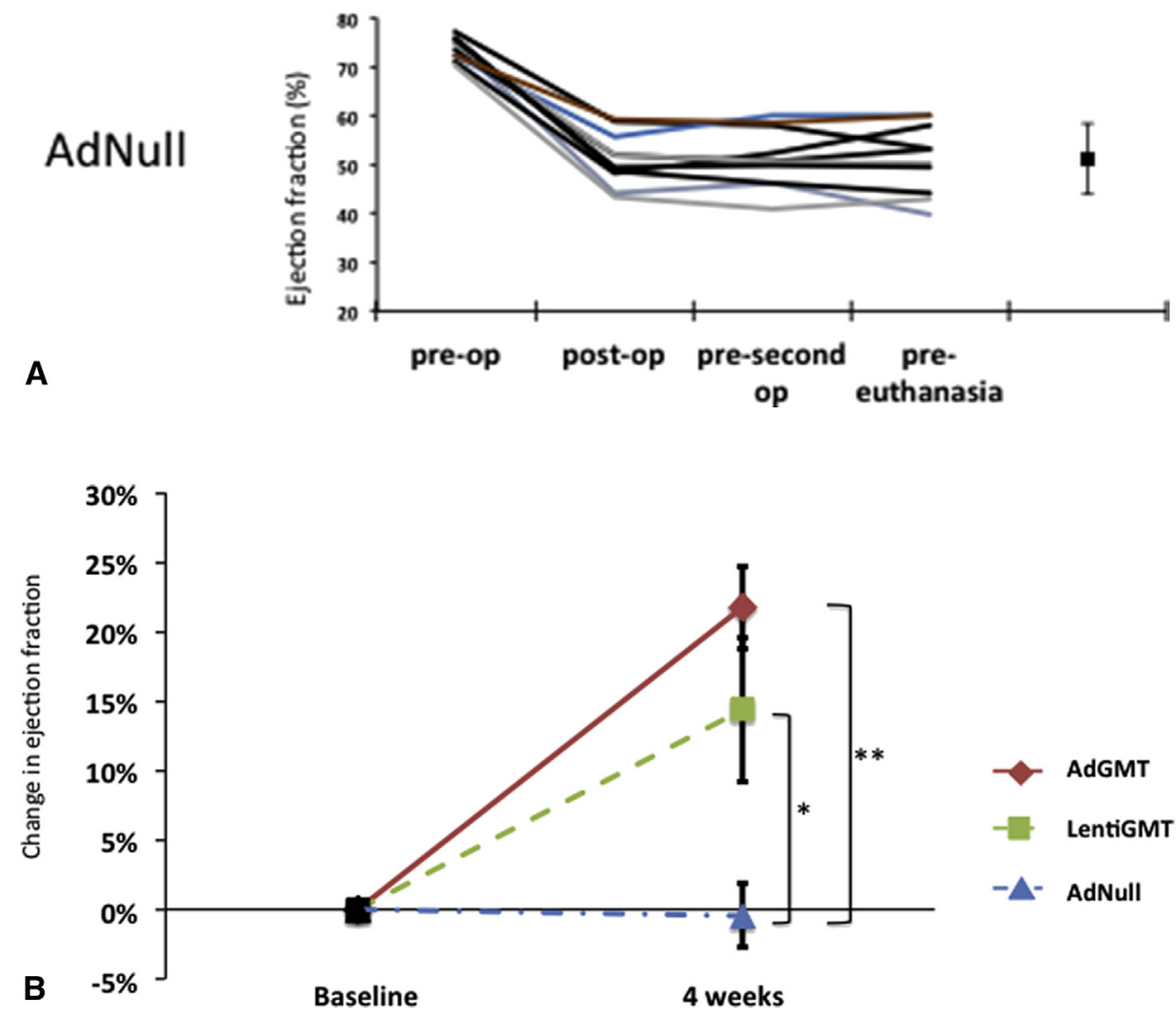

FIGURE 6. Improvements in ventricular function after administration of reprogramming factors in vivo. A, Global ejection fraction at specified time points for each animal obtained from echocardiographic studies performed, as described in the Methods, immediately before (pre-op) and 3 days after (post-op) coronary ligation and administration of AdVEGF, and immediately before (pre-second op) and 4 weeks after (pre-euthanasia) the administration of 
TABLE 1. Ejection fraction 4 weeks after reprogramming factor administration

\begin{tabular}{lcc}
\hline & MRI & Echocardiographic analysis \\
\hline AdGMT $(\mathrm{n}=11)$ & $60 \pm 2$ & $58 \pm 3$ \\
LentiGMT $(\mathrm{n}=10)$ & $57 \pm 3$ & $50 \pm 4$ \\
AdNull $(\mathrm{n}=10)$ & $56 \pm 2$ & $51 \pm 2$ \\
\hline
\end{tabular}

MRI, Magnetic resonance imaging; $A d G M T$, cocktail of adenovirus vectors expressing Gata4, Mef2c, or Tbx5; LentiGMT, cocktail of lentivirus vectors expressing gata4, Mef2c, or Tbx5; AdNull, adenoviral vector that does not encode a transgene.

In the context of these encouraging data regarding Ad-mediated cellular reprogramming, the widely held belief that Ad vector delivery will lead to systemic toxicity and reduced vector transduction must be considered. Little-to-no evidence of such toxicity actually exists, however, in the great many clinical trials that have used Ad vectors during the past 2 decades, including our own. ${ }^{34-36}$ This may be especially true for delivery such as in the current application using local administration, wherein vector cell entry occurs well before systemic immunity can develop. Although we did not observe any myocardial inflammation or other adverse effects in rats that had received $\mathrm{Ad}$, additional studies to clarify immunologic response are underway.

Abundant evidence from multiple previous in vitro and in vivo transgenic lineage-tracing murine studies supports our conclusion that the in situ reprogramming of cardiac fibroblasts into iCMs in regions of myocardial infarction is the basis of the improvements in hemodynamic function that we have observed in the postinfarct setting..$^{1-7,9-15}$ It is possible, however, that the in vivo improvements in postinfarct cardiac function observed in the current and previous studies are at least partially attributable to as-yet unidentified effects of reprogramming factors. For example, "bystander" effects on native cardiomyocytes could be produced through the ability of Ad vectors to infect both replicative (fibroblast) cells and nonreplicative (cardiomyocyte) cells.

Relative to mechanisms of action potentially responsible for our observed outcomes, it is important to note that the current studies include the administration of an Ad vector encoding VEGF at the time of coronary ligation to "prevascularize" the area of myocardial infarction before the administration of reprogramming vectors. This strategy is based on our previous demonstration that angiogenic pretreatment increases the observed density of implanted stem cells and/or iCMs and improved the hemodynamic outcomes in these studies. ${ }^{16,32}$ This is presumed to be attributable to the creation of a less ischemic milieu that better supports the survival of cardiomyocyte-like cells possessing greater metabolic rates and oxygen demands compared to resident scar fibroblasts. As in our previous studies, ${ }^{16,17,32}$ the absence of improved outcomes in the AdNull group in the current study allows us to ascribe these outcomes to the effects of reprogramming vector administration, rather than to any promiscuous effect of VEGF alone.

One limitation of the present study was our inability to reliably quantify $\mathrm{MYH}^{+}$cell numbers, particularly in the LentiGMT, although we have been able to do so previously. ${ }^{16}$ We were able to discern clear differences between the AdGMT and AdNull groups in $\mathrm{MYH}^{+}$cell density and have thus limited our reporting to semiquantitative description of these findings. Another limitation is the absence of lack of definitive evidence of the presumed fibroblast origin of the $\mathrm{MYH}^{+}$" $\mathrm{iCMs"}$ " we report in this study. In the absence of a readily available transgenic tag for such cells in the rat model, this conclusion is supported by the presence of dual vimentin/cTnT positive cells (Figure 5). We will be undertaking additional investigations to identify gap junction with connexin 43 and other markers of functional maturity in reprogrammed cells to further define this process.

In summary, the present study demonstrates that adenoviral vector encoding GMT can improve cardiac function after myocardial infarction in the rat heart. These findings suggest that adenoviral GMT administration is a potentially useful approach for the treatment of patients with heart failure after myocardial infarction.

\section{Conflict of Interest Statement}

Authors have nothing to disclose with regard to commercial support.

We thank Shuyun Deng, MD, from the Gene Vector Core at Baylor College of Medicine for the preparation of viral vectors, and Qianzi Zhang, MPH, for her help with the statistical analysis.

AdGMT, LentiGMT, or AdNull (AdGMT; $\mathrm{n}=11$, LentiGMT; $\mathrm{n}=10 ;$ AdNull; $\mathrm{n}=10$ ). Mean and standard deviation for ejection fraction 4 weeks after AdGMT, LentiGMT, and AdNull administration are depicted at right. B, Change in ejection fraction from the time of AdGMT, LentiGMT, and AdNull administration ( 3 weeks postcoronary ligation) to the time of follow-up echocardiography 4 weeks later (7 weeks after the coronary artery ligation) $* P<.05 ; * * P<.01$. LentiGMT signifies 3 singlets, LentiGata4, LentiMef2c, and LentiTbx 5 , and AdGMT signifies 3 singlets, AdGata4, AdMef2c, and AdTbx5. AdGMT, Cocktail of adenovirus vectors expressing Gata4, Mef2c, or Tbx5; LentiGMT, cocktail of lentivirus vectors expressing gata4, Mef2c, or Tbx5; AdNull, adenoviral vector that does not encode a transgene. 


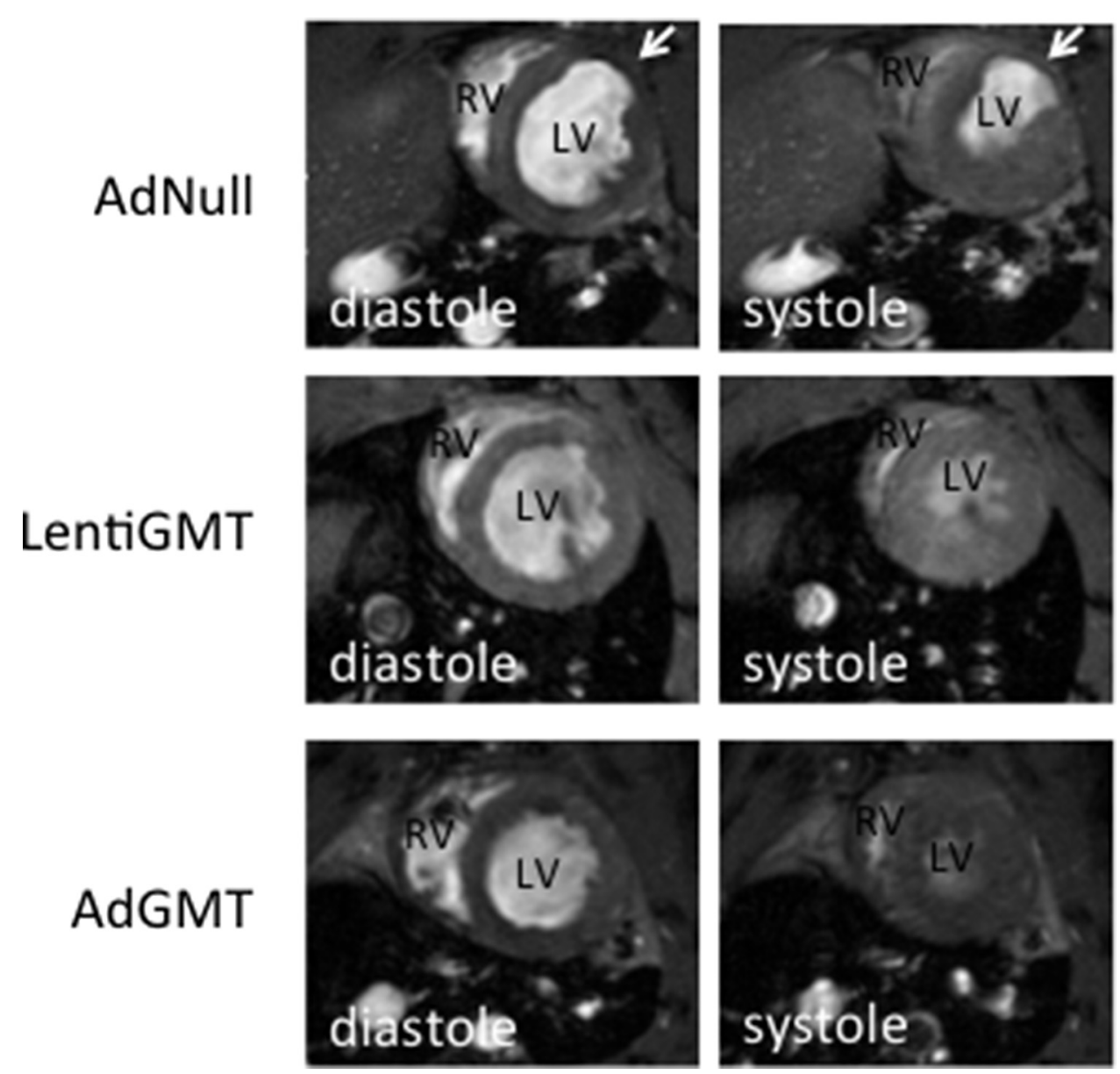

FIGURE 7. Representative MRI. Ejection fraction of the left ventricle was quantified by MRI 7 weeks after coronary artery ligation. The panels show representative transverse images of the thorax, containing hearts at the end of diastole (relaxation) or systole (contraction) from AdNull-, LentiGMT-, or AdGMT-injected rats. Arrows indicate thinning of the anterior wall in region of infarction created by coronary ligation in AdNull, but there is no thinning in GMT-treated animals. LentiGMT signifies 3 singlets, LentiGata4, LentiMef2c, and LentiTbx5, and AdGMT signifies 3 singlets, AdGata4, AdMef2c, and AdTbx5. $R V$, Right ventricle; $L V$, left ventricle; AdNull, adenoviral vector that does not encode a transgene; LentiGMT, cocktail of lentivirus vectors expressing gata4, Mef2c, or Tbx5; AdGMT, cocktail of adenovirus vectors expressing Gata4, Mef2c, or Tbx5.

\section{References}

1. Ieda M, Fu JD, Delgado-Olguin P, Vedantham V, Hayashi Y, Bruneau BG, et al. Direct reprogramming of fibroblasts into functional cardiomyocytes by defined factors. Cell. 2010;142:375-86.

2. Song K, Nam YJ, Luo X, Qi X, Tan W, Huang GN, et al. Heart repair by reprogramming non-myocytes with cardiac transcription factors. Nature. 2012; 485:599-604.

3. Jayawardena TM, Egemnazarov B, Finch EA, Zhang L, Payne JA, Pandya K, et al. MicroRNA-mediated in vitro and in vivo direct reprogramming of cardiac fibroblasts to cardiomyocytes. Circ Res. 2012;110: 1465-73.

4. Protze S, Khattak S, Poulet C, Lindemann D, Tanaka EM, Ravens U. A new approach to transcription factor screening for reprogramming of fibroblasts to cardiomyocyte-like cells. J Mol Cell Cardiol. 2012;53:323-32.

5. Fu JD, Stone NR, Liu L, Spencer CI, Qian L, Hayashi Y, et al. Direct reprogramming of human fibroblasts toward a cardiomyocyte-like state. Stem Cell Rep. 2013;1:235-47.

6. Nam YJ, Song K, Luo X, Daniel E, Lambeth K, West K, et al. Reprogramming of human fibroblasts toward a cardiac fate. Proc Natl Acad Sci USA. 2013;110: 5588-93.

7. Wada R, Muraoka N, Inagawa K, Yamakawa H, Miyamoto K, Sadahiro T, et al. Induction of human cardiomyocyte-like cells from fibroblasts by defined factors. Proc Natl Acad Sci USA. 2013;110:12667-72.
8. Takahashi K, Yamanaka S. Induction of pluripotent stem cells from mouse embryonic and adult fibroblast cultures by defined factors. Cell. 2006;126: 663-76.

9. Christoforou N, Chellappan M, Adler AF, Kirkton RD, Wu T, Addis RC, et al. Transcription factors MYOCD, SRF, Mesp1 and SMARCD3 enhance the cardio-inducing effect on GATA4, TBX5, and MEF2C during direct cellular reprogramming. PloS One. 2013;8:e63577.

10. Ifkovits JL, Addis RC, Epstein JA, Gearhart JD. Inhibition of TGF $\beta$ signaling increases direct conversion of fibroblasts to induced cardiomyocytes. PLoS One. 2014;9:e89678.

11. Muraoka N, Yamakawa H, Miyamoto K, Sadahiro T, Umei T, Isomi M, et al. MiR-133 promotes cardiac reprogramming by directly repressing Snail and silencing fibroblast signature. EMBO J. 2014;33:1565-81.

12. Wang L, Liu Z, Yin C, Asfour H, Chen OM, Li Y, et al. Stoichometry of Gata4, Mef2c, and Tbx5 influences the efficiency and quality of induced cardiac myocyte reprogramming. Circ Res. 2015;116:237-44.

13. Zhao Y, Londono P, Cao Y, Sharpe EJ, Proenza C, O'Rourke R, et al. High-efficiency reprogramming of fibroblasts into cardiomyocytes requires suppression of pro-fibrotic signaling. Nat Commun. 2015;6:8243.

14. Yamakawa H, Muraoka N, Miyamoto K, Sadahiro T, Isomi M, Haginiwa S, et al. Fibroblast growth factors and vascular endothelial growth factor promote cardiac reprogramming under defined conditions. Stem Cell Reports. 2015;5: $1128-42$. 
15. Zhou Y, Wang L, Vaseghi HR, Liu Z, Lu R, Alimohamadi S, et al. Bmi1 is a key epigenetic barrier to direct cardiac reprogramming. Cell Stem Cell. 2016;18:382-95.

16. Mathison M, Gersch RP, Nasser A, Lilo S, Korman M, Fourman M, et al. In vivo cardiac cellular reprogramming efficacy is enhanced by angiogenic preconditioning of the infarcted myocardium with vascular endothelial growth factor. J Am Heart Assoc. 2012;1:e005652.

17. Mathison M, Singh VP, Gersch RP, Ramirez MO, Cooney A, Kaminsky SM, et al. "Triplet" polycistronic vectors encoding Gata4, Mef2c, and Tbx5 enhances postinfarct ventricular functional improvement compared with singlet vectors. J Thorac Cardiovasc Surg. 2014;148:1656-64.e2.

18. Qian L, Huang Y, Spencer CI, Foley A, Vedantham V, Liu L, et al. In vivo reprogramming of murine cardiac fibroblasts into induced cardiomyocytes. Nature. 2012;485:593-8

19. Inagawa K, Miyamoto K, Yamakawa H, Muraoka N, Sadahiro T, Umei T, et al. Induction of cardiomyocyte-like cells in infarct hearts by gene transfer of Gata4, Mef2c, and Tbx5. Circ Res. 2012;111:1147-56.

20. Jayawardena TM, Finch EA, Zhang L, Zhang H, Hodgkinson CP, Pratt RE, et al. MicroRNA induced cardiac reprogramming in vivo Evidence for mature cardiac myocytes and improved cardiac function. Circ Res. 2015;116:418-24.

21. Hacein-Bey-Abina S, von Kalle C, Schmidt M, Le Deist F, Wulffraat N, McIntyre E, et al. A serious adverse event after successful gene therapy for X-linked severe combined immunodeficiency. N Engl J Med. 2003;348:255-6.

22. Yi Y, Noh MJ, Lee KH. Current advances in retroviral gene therapy. Curr Gene Ther. 2011;11:218-28.

23. Stadtfeld M, Maherali N, Breault DT, Hochedlinger K. Defining molecular cornerstones during fibroblast to iPS cell reprogramming in mouse. Cell Stem Cell. 2008;2:230-40.

24. Yang Y, Jooss KU, Su Q, Ertl HC, Wilson JM. Immune responses to viral antigens versus transgene product in the elimination of recombinant adenovirus-infected hepatocytes in vivo. Gene Ther. 1996;3:137-44.

25. Hackett NR, Kaminsky SM, Sondhi D, Crystal RG. Antivector and antitransgene host responses in gene therapy. Curr Opin Mol Ther. 2000;2:376-82.

26. Amano H, Hackett NR, Kaner RJ, Whitlock P, Rosengart TK, Crystal RG. Alteration of splicing signals in a genomic/cDNA hybrid VEGF gene to modify the ration of expressed VEGF isoforms enhances safety of angiogenic gene therapy. Mol Ther. 2005;12:716-24.
27. Rosenfeld MA, Yoshimura K, Trapnell BC, Yoneyama K, Rosenthal ER Dalemans W, et al. In vivo transfer of the human cystic fibrosis transmembrane conductance regulator gene to the airway epithelium. Cell. 1992;68:143-55.

28. Crabos M, Roth M, Hahn AW, Erne P. Characterization of angiotensin II receptors in cultured adult rat cardiac fibroblasts: coupling to signaling systems and gene expression. J Clin Invest. 1994;93:2372-8.

29. Lukasova M, Malaval C, Gille A, Kero J, Offermanns S. Nicotinic acid inhibit progression of atherosclerosis in mice through its receptor GPR109A expressed by immune cells. J Clin Invest. 2011;121:1163-73.

30. Crystal RG. Adenovirus: the first effective in vivo gene delivery vector. Hum Gene Ther. 2014;25:3-11.

31. Gilgenkrantz H, Duboc D, Juillard V, Couton D, Pavirani A, Guillet JG, et al Transient expression of genes transferred in vivo into heart using first-generation adenoviral vectors: role of the immune response. Hum Gene Ther. 1995;6:1265-74.

32. Wold WS, Toth K. Adenovirus vectors for gene therapy, vaccination and cancer gene therapy. Curr Gene Ther. 2013;13:421-33.

33. Sommer CA, Christodoulou C, Gianotti-Sommer A, Shen SS, Sailaja BS, Hezroni $\mathrm{H}$, et al. Residual expression of reprogramming factors affects the transcriptional program and epigenetic signatures of induced pluripotent stem cells. PLoS One. 2012;7:e51711.

34. Ahi YS, Bangari DS, Mittal SK. Adenoviral vector immunity: its implications and circumvention strategies. Curr Gene Ther. 2011;11:307-20.

35. Crystal RG, Harvey BG, Wisnivesky JP, O’Donoghue KA, Chu KW, Maroni J, et al. Analysis of risk factors for local delivery of low- and intermediate-dose adenovirus gene transfer vectors to individuals with a spectrum of comorbid conditions. Hum Gene Ther. 2002;13:65-100.

36. Retuerto MA, Schalch P, Patejunas G, Carbray J, Liu N, Esser K, et al Angiogenic pre-treatment improves the efficacy of cellular cardiomyoplasty performed with fetal cardiomyocyte implantation. J Thorac Cardiovasc Surg. 2004;127:1041-9.

Key Words: in situ cardiac reprogramming, adenoviral vector, gene therapy 

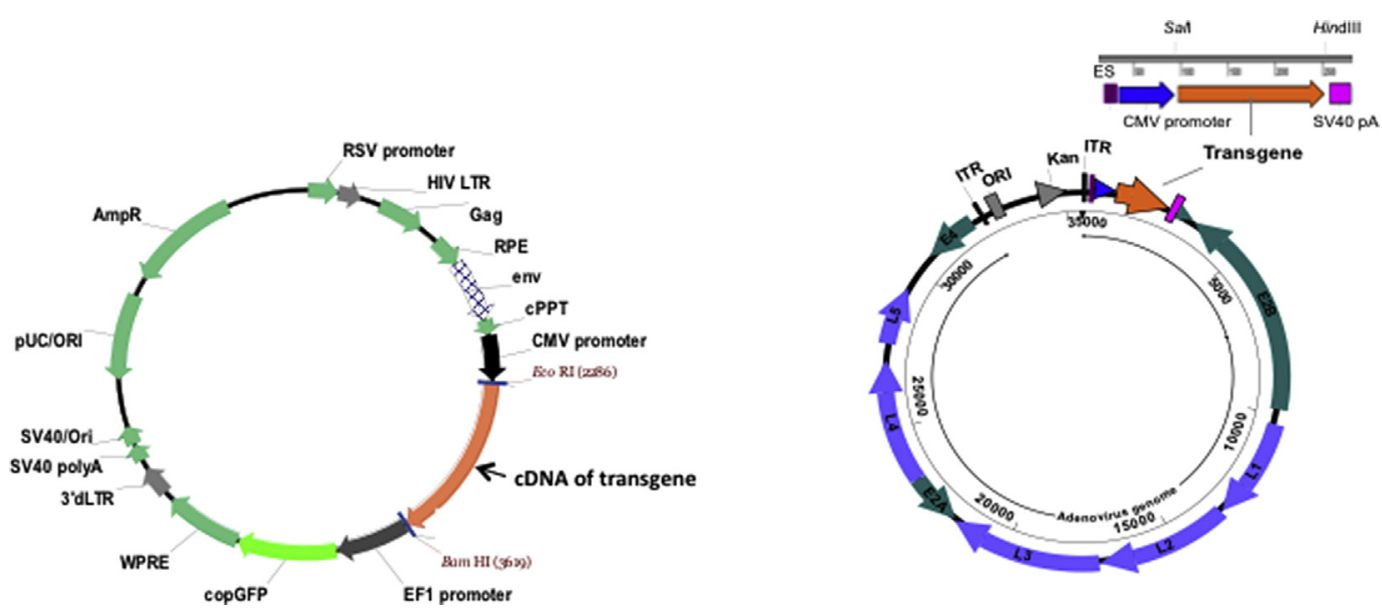

FIGURE E1. DNA construct maps for adenovirus and lentivirus vectors encoding Gata4, Mef2c, or Tbx5 transgenes. The lentiviral vectors (left) were constructed at BCM Gene Vector Core and the cDNA sequence for each transgene was subcloned into pCDH-CMV-EF1-GFP vector using EcoRI and HindIII restriction sites. The National Center for Biotechnology Information accession numbers for aforementioned cDNAs are NM_144730, XM_017594367, and NM_001009964, respectively. The adenoviral vectors (right) were constructed at the Belfer Gene Therapy Core Facility. The cDNAs were cloned first into the pShuttle-CMV plasmid (Agilent Technologies, Santa Clara, Calif) and then incorporated into the E1 region of the human adenovirus genome by homologous recombination with the RGD-modified pAdeasy (Agilent Technologies). The obtained pAd-G (or -M, -T) plasmids were used for adenoviral expression studies. RSV, Rous sarcoma virus; $L T R$, long terminal repeat; $R P E$, rev response element; $C M V$, cytomegalovirus; copGFP, Copepod Super Green Fluorescent Protein; WPRE, posttranscriptional regulatory element; SV40 polyA/SV40 pA, SV40 polyadenylation signal; ES, encapsidation signal; ITR, inverted terminal repeat; ORI, Escherichia coli origin of replication; Kan, kanamycin resistance gene; L1-L5, adenovirus late genes. 

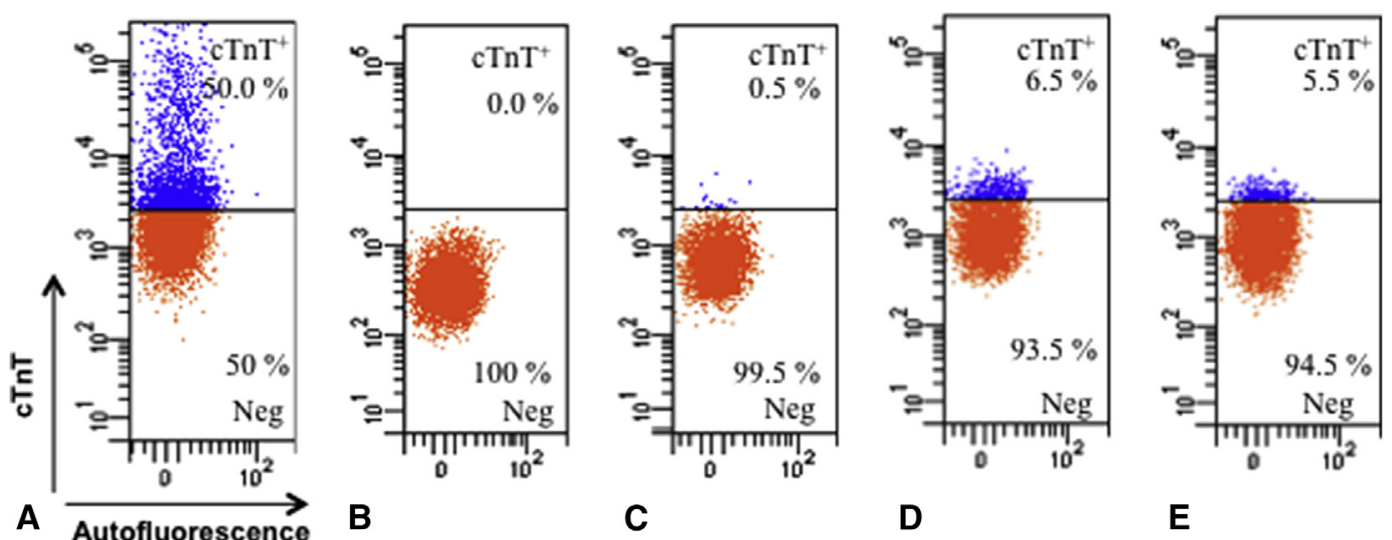

C

D

\section{$\mathbf{E}$}

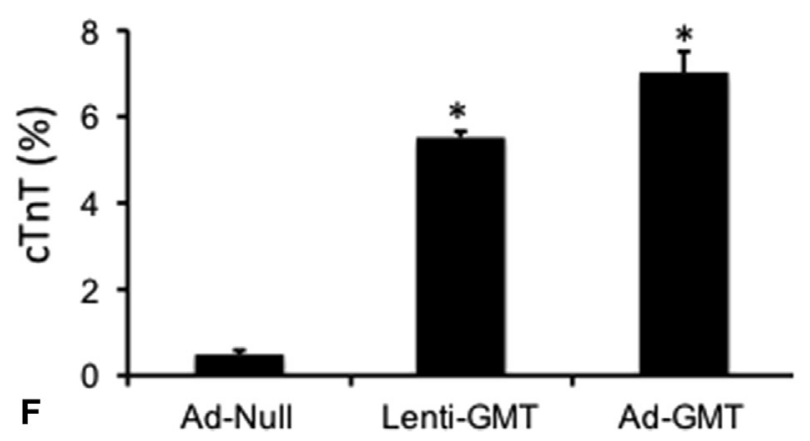

FIGURE E2. iCM transdifferentiation studies in vitro. Adult rat cardiac fibroblasts were infected in vitro with AdGMT, LentiGMT, or an AdNull control vector for 2 weeks, after which FACS sorting for the cardiomyocyte marker cTnT was performed, as described in the Methods. Representative FACS plots as follows. A, neonatal rat cardiac myocardium (positive control), B, secondary antibody alone, C, AdNull, D, AdGMT, or E, LentiGMT. Graphs show a minimum of 10,000 events. F, Summary of percentage of cells expressing cTnT as analyzed by FACS $(\mathrm{n}=3)$. $* P<.05$. LentiGMT signifies 3 singlets, LentiGata4, LentiMef2c, and LentiTbx5, and AdGMT signifies 3 singlets, AdGata4, AdMef2c, and AdTbx5. cTnT, Cardiac troponin; AdNull, adenoviral vector that does not encode a transgene; LentiGMT, cocktail of lentivirus vectors expressing gata4, Mef2c, or Tbx5; AdGMT, cocktail of adenovirus vectors expressing Gata4, Mef2c, or Tbx5.

\section{LentiGMT group}

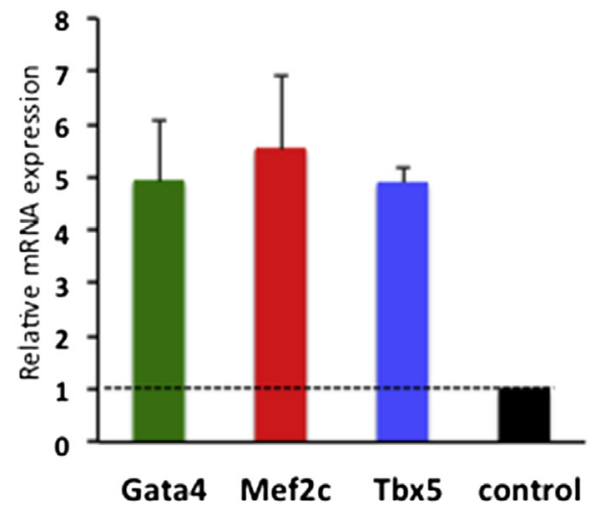

AdGMT group

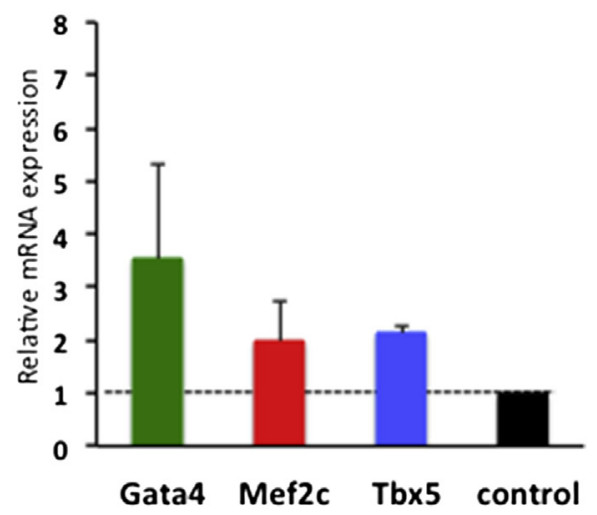

FIGURE E3. In vivo Gata4, Mef2c, and Tbx 5 transgene using adenovirus or lentivirus. qPCR of Gata4, Mef2c, and Tbx 5 on RNA extracted from the infarct area of hearts 7 weeks after MI and 4 weeks after injection of LentiGMT or negative control vector with an empty expression cassette. RNA was extracted 3 days after injection of AdGMT or AdNull. They are shown as relative to Control-injected rats, indicated by the dashed line. $\mathrm{N}=3$ for each group. LentiGMT signifies 3 singlets, LentiGata4, LentiMef2c, and LentiTbx5, and AdGMT signifies 3 singlets, AdGata4, AdMef2c, and AdTbx5. LentiGMT, Cocktail of lentivirus vectors expressing gata4, Mef2c, or Tbx5; AdGMT, cocktail of adenovirus vectors expressing Gata4, Mef2c, or Tbx5. 


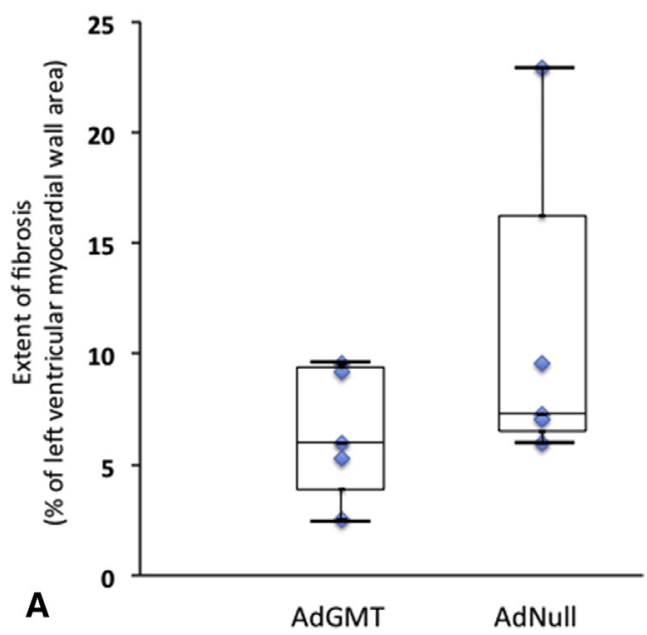

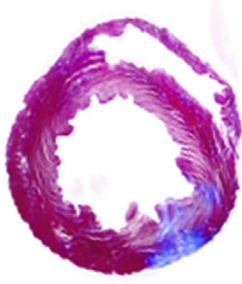

AdGMT

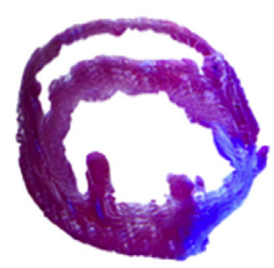

AdNull

FIGURE E4. Fibrosis assessment in postinfarct heart. Fibrosis area was measured with Masson-Trichrome staining, as described in the Methods. A, Average of extent of fibrosis ( $n=5$ per group). B, Representative photos of Masson-Trichrome staining. LentiGMT signifies 3 singlets, LentiGata4, LentiMef2c, and LentiTbx5, and AdGMT signifies 3 singlets, AdGata4, AdMef2c, and AdTbx5. AdGMT, Cocktail of adenovirus vectors expressing Gata4, Mef2c, or Tbx5; AdNull, adenoviral vector that does not encode a transgene. 